\title{
CT angiography vs echocardiography for detection of cardiac thrombi in ischemic stroke: a systematic review and meta-analysis
}

\author{
Nina-Suzanne Groeneveld ${ }^{1}$ - Valeria Guglielmi ${ }^{1} \cdot$ Mariska M. G. Leeflang $^{2} \cdot$ S. Matthijs Boekholdt ${ }^{3} \cdot$ R. Nils Planken ${ }^{4}$. \\ Yvo B. W. E. M. Roos ${ }^{1}$. Charles B. L. M. Majoie ${ }^{4}$. Jonathan M. Coutinho ${ }^{1}$ (])
}

Received: 29 November 2019 / Revised: 16 February 2020 / Accepted: 18 February 2020 / Published online: 5 March 2020

(c) The Author(s) 2020

\begin{abstract}
Background and purpose Cardiac thrombi are an important cause of embolic stroke. We studied the diagnostic yield and diagnostic accuracy of cardiac CT angiography (CTA) compared to echocardiography for detection of cardiac thrombi in ischemic stroke patients.

Methods We performed a systematic review and meta-analysis of the literature on cardiac CTA versus echocardiography for detection of cardiac thrombi in ischemic stroke patients. We included studies $(N \geq 20)$ in which both cardiac CTA (index test) and echocardiography (reference test) were performed and data on cardiac thrombi were reported. Results were stratified for type of echocardiography: transesophageal (TEE) vs transthoracic (TTE).

Results Out of 1530 studies, 14 were included (all single center cohort studies), with data on 1568 patients. Mean age varied between 52 and 69 years per study and $66 \%$ were men. Reported time intervals ranged from 0 to 21 days between stroke and first test, and from 0 to 199 days between tests. In ten studies that compared CTA to TEE, CTA detected cardiac thrombi in $87 / 1385(6.3 \%)$ patients versus 68/1385 $(4.9 \%)$ on TEE $(p<0.001)$. In four studies comparing CTA to TTE, CTA detected thrombi in $23 / 183(12.5 \%)$ patients versus $12 / 183(6.6 \%)$ on TTE $(p=0.010)$. Pooled sensitivity and specificity of CTA versus TEE were $86.0 \%$ (95\% CI 65.6-95.2) and 97.4\% (95\% CI 95.0-98.7), respectively.

Conclusions CTA may be a promising alternative to echocardiography for detection of cardiac thrombi in patients with ischemic stroke, especially now that CTA is standard care for patient selection for endovascular treatment. However, studies were too heterogeneous and of insufficient methodological quality to draw firm conclusions. Large, prospective studies on this topic are warranted.
\end{abstract}

Keywords Ischemic stroke $\cdot$ Cardiac emboli $\cdot$ Cardiac computed tomography $\cdot$ Echocardiography $\cdot$ Thrombus

Nina-Suzanne Groeneveld and Valeria Guglielmi have joint first authorship

Electronic supplementary material The online version of this article (https://doi.org/10.1007/s00415-020-09766-8) contains supplementary material, which is available to authorized users.

Jonathan M. Coutinho

j.coutinho@amsterdamumc.nl

1 Department of Neurology, Amsterdam UMC, University of Amsterdam, Amsterdam, The Netherlands

2 Department of Clinical Epidemiology and Biostatistics and Bioinformatics Amsterdam Public Health, Amsterdam UMC, University of Amsterdam, Amsterdam, The Netherlands

\section{Introduction}

Thromboembolism from a cardiac source accounts for up to one-third of all ischemic strokes [1-3]. Establishing cardioembolism is important in patients with ischemic stroke, since these patients are commonly managed with anticoagulation instead of antiplatelet therapy [4]. Thrombi in the

3 Department of Cardiology, Amsterdam UMC, University of Amsterdam, Amsterdam, The Netherlands

4 Department of Radiology and Nuclear Medicine, Amsterdam UMC, University of Amsterdam, Amsterdam, The Netherlands 
left atrial appendage (LAA) or left atrium (LA) secondary to atrial fibrillation (AF) are a major cause of cardioembolic stroke [4]. However, AF is often paroxysmal, which can be challenging to detect [5]. Moreover, conditions other than AF can also cause cardiac thrombi, such as thrombi in the left ventricle after myocardial infarction [4]. Timely detection of cardiac thrombi allows for early optimization of medical therapy.

Transthoracic echocardiography (TTE) and transesophageal echocardiography (TEE) are the first-line cardiac imaging modalities in stroke patients [6, 7]. Advantages are portable equipment, wide availability and relative inexpensiveness. TEE is more sensitive than TTE for detection of thrombi in LAA and LA [6, 7]. However, TEE is timeconsuming, burdensome for the patient, may require sedation, and has a low-but not negligible — risk of esophageal complications [6]. TTE is more suitable for evaluation of the left ventricle [7], but may be difficult in patients with poor acoustic windows, with obesity or breast implants, and in those suffering from chronic obstructive pulmonary disease. The quality of echocardiography is also dependent on operator expertise. Cardiac CT angiography (CTA) is an alternative imaging technique for detection of cardiac thrombi. It may be a particularly useful modality in acute ischemic stroke patients, now that CTA of the cervical and intracranial arteries has become routine care to determine eligibility for endovascular treatment and cardiac CTA could be included in the same imaging protocol [8-10]. Furthermore, the additional radiation associated with cardiac CTA is low with modern CT scanners [11]. Our aim was to study the diagnostic yield and diagnostic accuracy of CTA for detection of cardiac thrombi in stroke patients, compared to echocardiography, by performing a systematic review and meta-analysis of the literature.

\section{Methods}

\section{Study design}

We searched Medline and Excerpta Medica database (EMBASE) from inception to June 7th 2019 using the following search terms: ischemic stroke, cardiac CT angiography, and cardiac thrombus (Online Resource Table I). Rayyan (https://rayyan.qcri.org) was used for study screening [12]. Title and abstract screening was performed independently by two authors (NSG and VG). Full-length review of potentially relevant studies and conference abstracts was performed by one author (NSG). We also screened reference lists of included articles to identify additional potentially relevant studies. Final decisions regarding study selection were reached through consensus. We registered the protocol in the International Prospective Register of Systematic
Reviews (PROSPERO), IC CRD42018103658. We wrote our report according to Preferred Reporting Items for Systematic Reviews and Meta-Analyses (PRISMA) guidelines for Diagnostic Test Accuracy (DTA) studies [13].

\section{Inclusion and exclusion criteria}

We included studies in any language that: (1) performed cardiac CTA as well as echocardiography (TEE or TTE); (2) in patients with ischemic stroke or transient ischemic attack (TIA); (3) allowed data extraction on cases with cardiac thrombi; (4) had a randomized controlled trial or a cross-sectional diagnostic accuracy study design (cohort or case control with a minimum population size of 20 patients). Abstracts containing sufficient data were also included. In case of overlapping patient cohorts between studies, the study with the largest sample size was included.

\section{Index test and reference standard, study endpoints}

A true gold standard for detection of cardiac thrombi is lacking. In our study, cardiac CTA was considered the index test and echocardiography the reference standard. The decision to use echocardiography as the reference test was based on the fact that international guidelines recommend echocardiography as first-line screening method for imaging of the heart in ischemic stroke patients [6,7]. Results were stratified for type of echocardiography: CTA versus TEE and CTA versus TTE. Studies in which patients underwent both TTE and TEE, but only TEE results were clearly reported, were considered as CTA versus TEE. The primary endpoint was diagnostic yield, defined as proportion of patients with a cardiac thrombus on CTA or echocardiography. The secondary endpoint was diagnostic accuracy, defined as sensitivity and specificity of cardiac CTA compared to echocardiography. Due to the lack of a true gold standard, diagnostic yield and not diagnostic accuracy was chosen as primary endpoint.

\section{Quality assessment and data extraction}

Both quality assessment and data extraction were independently performed by two authors (N.S.G. and V.G.). In situations of disagreement, a third author made the final decision (M.M.G.L. for quality assessment and J.M.C for data extraction). The Quality Assessment of Diagnostic Accuracy Studies (QUADAS)-2 checklist was used to assess risk of bias and concerns regarding applicability [14]. The different components of the QUADAS-2 guidelines were tailored to our research question (Online Resource Tables IIa-IIb).

We extracted data on study characteristics (study design, publication year, sample size) and study population characteristics (mean age, sex, ischemic stroke or TIA, presence or absence of cardiac medical history/disease). We also 
collected data on CTA and echocardiography techniques, such as type of scanner, ultrasound frequency, cardiac imaging protocols, definition of cardiac thrombus, order of testing, time interval between stroke onset and first test, time interval between tests, and amount of cardiac thrombi found through cardiac CTA and echocardiography. We collected data on thrombi detected in any location in the heart (LAA, LA, left ventricle, right atrium, right ventricle).

\section{Statistical analysis}

To determine differences in diagnostic yield, the OpenEpi ${ }^{\circledR}$ software program version 3.01 (Open Source Epidemiologic Statistics for Public Health) was used to perform a McNemar test for paired data. Data of patients who underwent both CTA and TEE, and CTA and TTE, respectively, were analyzed using a two-sided McNemar test with binomial enumeration (Mid-P Exact) and a significance level of 0.05 . To study diagnostic accuracy, sensitivity and specificity of CTA versus TEE and CTA versus TTE were calculated for all studies based on reported cardiac thrombus cases. Forest plots were generated using Review Manager version 5.3 (RevMan, Copenhagen: The Nordic Cochrane Centre, The Cochrane Collaboration, 2014). Studies for which both sensitivity and specificity were estimable were included in a meta-analysis. Summary receiver operating curves (SROC) with estimated area under the curve (AUC) and pooled sensitivity and specificity were generated using $\mathrm{R}$ version 3.2.5 (R Foundation for Statistical Computing, Vienna, Austria) using the Reitsma bivariate mixed effects model incorporated in the mada package version 0.5.8. Pooled sensitivity and specificity were also generated for a subgroup of studies that used 64-slice CT scanners or newer generations and for studies in which the reported time interval between CTA and echocardiography was approximately 1 day (mean/median/ absolute amount of up to 1 day).

\section{Results}

\section{Study selection}

A flowchart of the study selection is provided in Fig. 1. We identified 1530 publications in the initial search, of which 42 were selected for full-length review. Of these, 14 articles fulfilled the inclusion criteria and were included in the analyses [8, 15-27].

\section{Study quality}

Overall, the risk of bias was highest for the domains 'patient selection' and 'reference standard' (high or unclear risk of bias in 5/14 (35.7\%) and 6/14 (42.9\%) studies, respectively) (Online Resource Figure Ia and Ib). Regarding the domain 'patient selection', studies did not describe clearly why and/ or how many patients were excluded. Regarding the domain 'reference standard', studies were unclear about whether the reference standard results were interpreted without knowledge of the results of the index test. In addition, for both the index and reference tests, there were issues with lack of reporting of the definition of a cardiac thrombus. Ten out of $14(71.4 \%)$ studies defined cardiac thrombus on CTA and 8/14 (57.1\%) on echocardiography. Only 2 out of 14 $(14.3 \%)$ studies had a low risk of bias for all of the domains of the QUADAS-2 checklist [16, 18]. Concerns regarding applicability were generally low except for the domain
Fig. 1 Flowchart of study selection. TIA indicates transient ischemic attack; RCT, randomized controlled trial. For detailed data of excluded studies please see Online Resource Reference list of excluded studies, appendix to Fig. 1

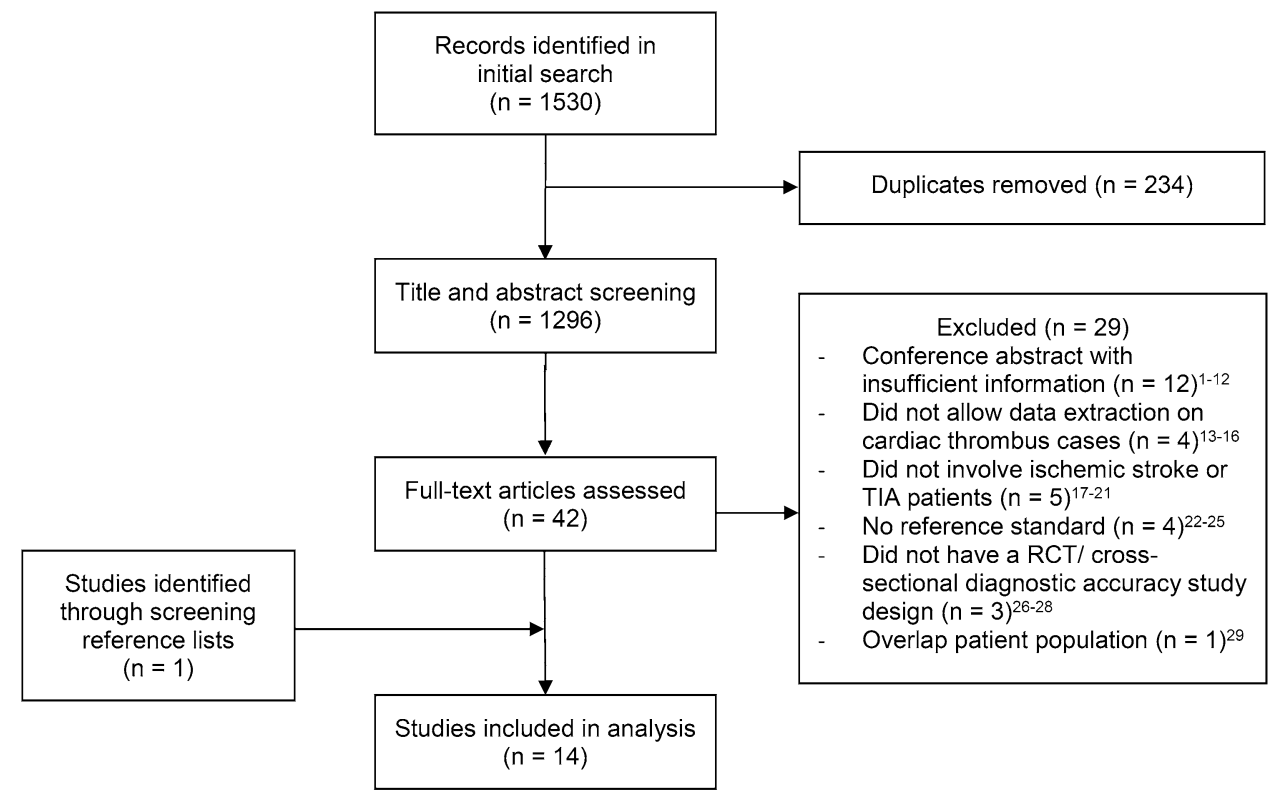


'interpretation and conduct of the index-test' for which two studies scored a high concern due to using outdated CT scanners (Imatron C-100) [15, 16].

\section{Study characteristics}

The study characteristics of the 14 included studies are shown in Table 1. All studies were single center cohort studies (12 prospective and 2 retrospective), published between 1989 and 2017. In total, data of 1568 patients were available. Sample sizes of individual studies ranged from 20 to 374 patients.

Eleven of 14 studies were conducted exclusively in ischemic stroke patients, while three studies also included TIA patients. Four studies had a general ischemic stroke population regardless of medical history or stroke etiology, seven studies included ischemic stroke patients with a suspected cardioembolic source, two studies included patients with a suspected cardioembolic source other than AF, and one study excluded patients with a probable cardioembolic source. Overall, 308/1532 (20.1\%) of patients for which this data were available had AF.

Details on CTA and echocardiography methods are reported in Table 2. Ten studies compared performance of CTA to TEE, four studies compared CTA to TTE. The order of tests was reported in 9/14 (64.3\%) studies. Four studies performed cardiac CTA first, four echocardiography first, and in one study the order of tests varied. Time interval between stroke onset and first test was reported in 8/14 (57.1\%) studies and was within 2 weeks for most of these studies. Time interval between tests was reported in 13/14 (92.8\%) studies and ranged between 0 and 199 days. In most studies, the second test was done within 1 week after the first, four studies reported a time interval of approximately 1 day. Only one study performed cardiac CTA in the acute phase of ischemic stroke [8], i.e. within the time window for acute reperfusion therapy (intravenous thrombolysis and/or endovascular treatment). This study performed non ECGgated CTA of the entire heart-brain axis in acute ischemic stroke patients within $4.5 \mathrm{~h}$ of symptom onset. For cardiac CTA, prospective ECG gating (when the scanner is triggered to scan the heart only during a predetermined phase of the cardiac cycle) was used in three studies, retrospective ECG gating (when all phases of the heart are scanned and images of the phase of interest are selected retrospectively) was used in eight studies, one study used both techniques and two studies did not use ECG registration. Nine studies used 64-slice CT or newer generation scanners, two studies used dual-source scanners.

\section{Diagnostic yield}

Data on detection of thrombi are provided in Table 3 (CTA versus TEE) and Table 4 (CTA versus TTE). In 1385 patients who underwent CTA and TEE, CTA detected cardiac thrombi in $87(6.3 \%)$ patients compared to in $68(4.9 \%)$

Table 1 Study design and patient characteristics

\begin{tabular}{|c|c|c|c|c|c|}
\hline References & Study design & $\begin{array}{l}\text { Num- } \\
\text { ber of } \\
\text { patients }\end{array}$ & $\begin{array}{l}\text { Mean age in } \\
\text { years (SD or } \\
\text { range) }\end{array}$ & Men $(\%)$ & Population characteristics I proportion of patients with AF (\%) \\
\hline Helgason [15] & Prospective cohort & 38 & $61(18-86)$ & NR & Stroke of suspected cardioembolic source | 7/38 (18.4) \\
\hline Love [16] & Prospective cohort & 36 & $60(\mathrm{NR})$ & $20(56)$ & Stroke or TIA of suspected cardioembolic source I NR \\
\hline Hur [17] & Retrospective cohort & 101 & $69(45-81)$ & $63(62)$ & Stroke of suspected cardioembolic source | 21/101 (20.8) \\
\hline Hur [18] & Prospective cohort & 137 & $61(13)$ & $95(69)$ & Stroke of suspected cardioembolic source | 57/137 (41.6) \\
\hline Boussel [19] & Prospective open-pilot & 39 & $63(11)$ & $38(83)$ & Stroke I 3/39 (7.7) \\
\hline Kim [20] & Prospective cohort & 314 & $65(13)$ & $186(59)$ & Stroke of suspected cardioembolic source I 72/314 (22.9) \\
\hline Ko [21] & Prospective cohort & $75^{*}$ & $67(58-72)$ & $42(56)$ & Stroke | 48/143 (33.6) \\
\hline Hur [22] & Prospective comparison & 83 & $63(36-83)$ & $56(67)$ & Stroke of suspected cardioembolic source I 49/83 (59.0) \\
\hline Kim [23] & Prospective cohort & $62^{*}$ & $68(60-74)$ & $46(74)$ & Stroke without AF and without cardiac disease I 0/62 (0.0) \\
\hline Sipola $[24]$ & Prospective cohort & 140 & $60(10)$ & $95(68)$ & $\begin{array}{l}\text { Stroke or TIA of suspected cardioembolic source without AF } \\
\text { । } 0 / 140(0.0)\end{array}$ \\
\hline Lee [25] & Prospective cohort & 374 & $63(20-89)$ & $254(68)$ & Stroke | 45/374 (12.0) \\
\hline Ajlan [26] & Retrospective cohort & 47 & $52(11)$ & $25(53)$ & Stroke of suspected cardioembolic source I 2/47 (4.3) \\
\hline Taina [27] & Prospective cohort & 102 & $62(11)$ & $70(69)$ & $\begin{array}{l}\text { Stroke or TIA of suspected cardioembolic source without AF } \\
\text { । } 0 / 102(0.0)\end{array}$ \\
\hline Yeo [8] & Prospective open-pilot & 20 & $64(12)$ & $13(65)$ & Stroke patients eligible for reperfusion therapy | 4/20 (20.0) \\
\hline
\end{tabular}

$A F$ atrial fibrillation, $N R$ not reported, Stroke ischemic stroke, TIA transient ischemic attack

*The study was divided into three different periods/groups. Only the period/group in which patients were assessed with both echocardiography and $\mathrm{CT}$ is included in this table 
Table 2 CT and echocardiography methods per study

\begin{tabular}{|c|c|c|c|c|c|}
\hline References & $\begin{array}{l}\text { First test: time interval } \\
\text { between stroke and first test } \\
\text { in days }\end{array}$ & $\begin{array}{l}\text { Reported time interval } \\
\text { between tests in days }\end{array}$ & TEE/TTE: MHz & CT type slice & $\begin{array}{l}\text { Cardiac CTA ECG gating } \\
\text { technique }\end{array}$ \\
\hline Helgason [15] & $\mathrm{CT}: \leq 21$ & $<1$ & TTE: NR & Imatron $\mathrm{C}-100$ & Retrospective \\
\hline Love $[16]$ & CT: range $0-13$ & Mean 3.5 (range 0-7) & TTE: $2.5 / 3.5$ & Imatron C-100 & No ECG gating \\
\hline Hur [17] & TEE: $<14$ & $<7$ & TEE: 5 & $64 \mathrm{~s}$ & Retrospective \\
\hline Hur [18] & TEE: $<7$ & Mean 5 (SD 2.8) & TEE: 5 & $64 \mathrm{~s}$ & Retrospective \\
\hline Boussel [19] & NR & $\begin{array}{l}\text { TTE: mean } 0.8 \text { (SD 2.4) } \\
\text { TEE: mean } 2.5 \text { (SD 2.8) }\end{array}$ & $\begin{array}{l}\text { TTE: } 2.5-3.5 \\
\text { TEE: } 5\end{array}$ & $40 \mathrm{~s}$ & Retrospective \\
\hline Kim [20] & NR & $<7$ & TEE: 7 & $64 s$ & Prospective \\
\hline Ko [21] & NR & Median 4 & $\begin{array}{l}\text { TTE: } 3.5 \\
\text { TEE: } 7\end{array}$ & $64 \mathrm{~s}$ & Retrospective \\
\hline Hur [22] & TEE: mean 6.8 (range 5-13) & Mean 2.3 & TEE: 5-7 & $128 \mathrm{~s}$ dual source & Prospective \\
\hline Kim [23] & NR & NR & TTE: 2.5 & $64 \mathrm{~s}$ & Retrospective \\
\hline Sipola [24] & CT: mean 6 (SD 4) & $<1$ & $\begin{array}{l}\text { TTE: } 3 \\
\text { TEE: } 6\end{array}$ & $16 \mathrm{~s}$ and $64 \mathrm{~s}$ & Retrospective \\
\hline Lee [25] & TEE: $<7$ & Mean 4.64 & TEE: $5-7$ & $64 \mathrm{~s}$ and $128 \mathrm{~s}$ & Prospective \\
\hline Ajlan [26] & TTE/CT: NR & Mean 19 (range 0-199)* & TTE: (NR) & $128 \mathrm{~s}$ dual source & Prospective + retrospective \\
\hline Taina [27] & NR & Mean $0.92(\mathrm{SD} 4.45)$ & TEE: 5-7 & $16 \mathrm{~s}$ & Retrospective \\
\hline Yeo $[8]$ & CT: $<1$ & $<1$ & $\begin{array}{l}\text { TTE: } 2.5-3.5 \\
\text { TEE: } 5\end{array}$ & $64 \mathrm{~s}$ & No ECG gating \\
\hline
\end{tabular}

$A F$ atrial fibrillation, $C T A$ computed tomography angiography, $M H z$ megahertz, $N R$ data not reported, $S D$ standard deviation, $T E E$ transesophageal echocardiography, TIA transient Ischemic Attack, TTE transthoracic echocardiography

*Based on data from 34/47 patients

Table 3 Patients with cardiac thrombi detected on CT angiography (CTA) versus transesophageal echocardiography (TEE)

\begin{tabular}{llllll}
\hline References & $N$ & $\begin{array}{l}\text { Patients with } \\
\text { thrombi on } \\
\text { CTA }\end{array}$ & $\begin{array}{l}\text { Patients with } \\
\text { thrombi on } \\
\text { TEE }\end{array}$ & $\begin{array}{l}\text { Patients with thrombi } \\
\text { on CTA but not on TEEE }\end{array}$ & $\begin{array}{l}\text { Patients with thrombi } \\
\text { on TEE but not on } \\
\text { CTA }\end{array}$ \\
\hline Hur [17] & 101 & 12 & 8 & 4 & 0 \\
Hur [18] & 137 & 12 & 12 & 0 & 0 \\
Boussel [19] & 39 & 1 & 0 & 1 & 0 \\
Kim [20] & 314 & 29 & 23 & 6 & 0 \\
Ko [21] & 75 & 8 & 1 & 7 & 0 \\
Hur [22] & 83 & 13 & 13 & 0 & 0 \\
Sipola [24] & 140 & 1 & 3 & 1 & 3 \\
Lee [25] & 374 & 6 & 6 & 0 & 0 \\
Taina [27] & 102 & 3 & 0 & 3 & 0 \\
Yeo [8] & 20 & 2 & 2 & 0 & 0 \\
Total & 1385 & $87(6.3 \%)$ & $68(4.9 \%)$ & $22(1.6 \%)$ & $3(0.2 \%)$ \\
\hline
\end{tabular}

$N$ indicates the number of patients with ischemic stroke who underwent both CTA and TEE patients on TEE $(p<0.001)$. In a total of $22 / 1385(1.6 \%)$ patients, thrombi were detected on CTA but not on TEE, while in $3 / 1385(0.2 \%)$ patients thrombi were detected on TEE but not on CTA. Location of cardiac thrombi detected on CTA was LAA in 68/87 (78.2\%), LA in 2/87 (2.3\%), left ventricle in $3 / 87$ (3.4\%) and not specified in 14/87 (16.1\%) patients. Location of cardiac thrombi detected on TEE was
LAA in 56/68 (82.4\%), LA in 4/68 (5.9\%), left ventricle in $1 / 68(1.5 \%)$ and not specified in $7 / 68(10.3 \%)$ patients.

In 183 patients who underwent CTA and TTE, CTA detected thrombi in $24(13.1 \%)$ patients compared to in $12(6.6 \%)$ on TTE $(p=0.010)$. In $18 / 183(9.8 \%)$ patients, thrombi were detected on CTA but not on TTE, in 7/183 (3.8\%) patients thrombi were detected on TTE but not on CTA. Location of cardiac thrombi detected on CTA was 
Table 4 Patients with cardiac thrombi detected on $\mathrm{CT}$ angiography (CTA) versus transthoracic echocardiography (TTE)

\begin{tabular}{llllll}
\hline References & $N$ & $\begin{array}{l}\text { Patients with } \\
\text { thrombi on } \\
\text { CTA }\end{array}$ & $\begin{array}{l}\text { Patients with } \\
\text { thrombi on } \\
\text { TTE }\end{array}$ & $\begin{array}{l}\text { Patients with thrombi } \\
\text { on CTA but not on TTE }\end{array}$ & $\begin{array}{l}\text { Patients with thrombi } \\
\text { on TTE but not on } \\
\text { CTA }\end{array}$ \\
\hline Helgason [15] & 38 & 11 & 2 & $11^{*}$ & 2 \\
Love [16] & 36 & 6 & 10 & 1 & 5 \\
Kim [23] & 62 & 0 & 0 & 0 & 0 \\
Ajlan [26] & 47 & 6 & 0 & 6 & 0 \\
Total & 183 & $23(12.5 \%)$ & $12(6.6 \%)$ & $18(9.8 \%)$ & $7(3.8 \%)$ \\
\hline
\end{tabular}

$N$ indicates the number of patients with ischemic stroke who underwent both CTA and TTE

*In this study CTA detected two thrombi in one patient
LAA in $2 / 24(8.3 \%)$, LA in $9 / 24(37.5 \%)$, left ventricle in $7 / 24(29.2 \%)$ and not specified in $6 / 24(25.0 \%)$ patients. Location of cardiac thrombi detected on TTE was LA in $2 / 12(16.7 \%)$ and not specified in 10/12 (83.3\%).

\section{Diagnostic accuracy}

Sensitivity of cardiac CTA with reference standard TEE was estimable in $8 / 10$ studies and was $100 \%$ in $7 / 8$ and $0 \%$ in $1 / 8$ studies (Fig. 2). Specificity was calculated in $10 / 10$ TEE studies and ranged between 91 and $100 \%$. The pooled sensitivity of CTA compared to TEE was $86.0 \%$ (95\% CI 65.6-95.2\%) and the pooled specificity was $97.4 \%$ (95\% CI 95.0-98.7\%) (Online Resource Figure IIa).

Sensitivity of cardiac CTA with reference standard TTE was estimable in $2 / 4$ studies and was $0 \%$ and $50 \%$, respectively. Specificity was calculated in 4/4 TTE studies and ranged between 69 and $100 \%$. The pooled sensitivity of
CTA compared to TTE was $37.0 \%$ (95\% CI 12.6-70.4\%), the pooled specificity was $83.9 \%$ (95\% CI 63.4-94.0\%) (Online Resource Figure IIb).

The pooled sensitivity of CTA compared to TEE in the 7/10 CTA studies which used 64-slice CT scanners or newer generations was $93.7 \%$ (95\% CI 82.6-97.9\%) and the pooled specificity was $96.7 \%$ (95\% CI 93.8-99.2\%). After excluding two studies which used outdated CT scanners, there were insufficient data to perform a pooled analysis for sensitivity and specificity of CTA versus TTE.

The pooled sensitivity of CTA compared to TEE in the $4 / 10$ studies in which the time interval between the two investigations was approximately 1 day was $47.7 \%$ (95\% CI 7.7-90.9\%) and the pooled specificity was $97.6 \%$ (95\% CI 93.4-99.1\%). After excluding two studies with a time interval exceeding 1 day and one with an unreported time interval, there were insufficient data to perform a pooled analysis for sensitivity and specificity of CTA versus TTE.

CT-angiography (CTA) versus trans esophageal echocardiography (TEE)

\begin{tabular}{|c|c|c|c|c|c|c|c|c|c|}
\hline Study & $\mathrm{TP}$ & FP & $\mathrm{FN}$ & TN & Sample size & Sensitivity $(95 \% \mathrm{Cl})$ & Specificity $(95 \% \mathrm{Cl})$ & Sensitivity $(95 \% \mathrm{Cl})$ & Specificity $(95 \% \mathrm{Cl})$ \\
\hline Hur J. et al (2008) & 8 & 4 & 0 & 89 & 101 & $1.00[0.63,1.00]$ & $0.96[0.89,0.99]$ & & \\
\hline Hur J. et al (2009), Stroke & 12 & 0 & 0 & 125 & 137 & $1.00[0.74,1.00]$ & $1.00[0.97,1.00]$ & & \\
\hline Boussel L. et al (2010) & 0 & 1 & 0 & 38 & 39 & Not estimable & $0.97[0.87,1.00]$ & & $\rightarrow$ \\
\hline Ko SB. et al $(2010)$ & 1 & 7 & 0 & 67 & 75 & $1.00[0.03,1.00]$ & $0.91[0.81,0.96]$ & & $\rightarrow$ \\
\hline Kim SC. et al (2010) & 23 & 6 & 0 & 285 & 314 & $1.00[0.85,1.00]$ & $0.98[0.96,0.99]$ & & - \\
\hline Hur J. et al (2011) & 13 & 0 & 0 & 70 & 83 & $1.00[0.75,1.00]$ & $1.00[0.95,1.00]$ & & \\
\hline Sipola P. et al (2013) & 0 & 1 & 3 & 136 & 140 & $0.00[0.00,0.71]$ & $0.99[0.96,1.00]$ & & \\
\hline Lee K. et al (2015) & 6 & 0 & 0 & 368 & 374 & $1.00[0.54,1.00]$ & $1.00[0.99,1.00]$ & & \\
\hline Taina M. et al (2016) & 0 & 3 & 0 & 99 & 102 & imable & 0.99] & & \\
\hline Yeo L. et al (2017) & 2 & 0 & 0 & 18 & 20 & $1.00[0.16,1.00]$ & $1.00[0.81,1.00]$ & & \\
\hline \multirow{2}{*}{\multicolumn{10}{|c|}{ CT-angiography (CTA) versus transthoracic echocardiography (TTE) }} \\
\hline & & & & & & & & & \\
\hline Study & TP & $\mathrm{FP}$ & $\mathrm{FN}$ & TN & Sample size & Sensitivity $(95 \% \mathrm{Cl})$ & Specificity (95\% Cl) & Sensitivity $(95 \% \mathrm{Cl})$ & Specificity $(95 \% \mathrm{Cl})$ \\
\hline Helgason CM. et al & 0 & 11 & 2 & 24 & 38 & $0.00[0.00,0.84]$ & $0.69[0.51,0.83]$ & & \\
\hline Love BB. et al (1990) & 5 & 1 & 5 & 25 & 36 & $0.50[0.19,0.81]$ & $0.96[0.80,1.00]$ & & \\
\hline Kim S.J. et & 0 & 0 & 0 & 62 & 62 & Not estimable & $1.00[0.94,1.00]$ & & \\
\hline Ajlan A. et al (2016) & 0 & 6 & 0 & 41 & 47 & Not estimable & $0.87[0.74,0.95]$ & & $\rightarrow$ \\
\hline
\end{tabular}

Fig. 2 Sensitivity and specificity of cardiac CT angiography compared to echocardiography for detection of cardiac thrombus in stroke patients. $F N$ false negative, $F P$ false positive, $T N$ true negative, $T P$ true positive 


\section{Discussion}

In our systematic review, we used data of 1568 ischemic stroke patients to determine the diagnostic yield of CTA and echocardiography for detection of cardiac thrombi. Our results suggest that cardiac thrombi are more often detected on cardiac CTA than on TEE or TTE. When examined in terms of diagnostic accuracy, CTA appears to have high sensitivity and specificity compared to TEE, while there was insufficient data for conclusions on sensitivity and specificity for CTA versus TTE. A previous meta-analysis found that cardiac CTA is a reliable alternative to TEE for detection of left atrium and left atrial appendage thrombi [28]. Our study distinguishes itself by focusing specifically on the ischemic stroke/TIA population, reporting also on left ventricular thrombi and the diagnostic yield of CTA versus TTE, and incorporating recent studies.

The methodological quality of studies was low in many aspects. Only $2 / 14$ studies had a low risk of bias on all domains of the QUADAS-2 checklist [16, 18]. In addition, the definition of cardiac thrombus on CTA and echocardiography was often not reported and varied, which could have led to reporting bias. Furthermore, study populations were quite heterogeneous, which could have influenced the results. As expected, a larger proportion of cardiac thrombi was found in study populations with suspected cardioembolic source, followed by general ischemic stroke populations. In the study of Kim et al. [23], patients with AF or any history of cardiac disease were excluded and no cardiac thrombi were found, despite the fact that this study contained the largest patient population for studies comparing CTA to TTE.

Time interval between stroke and first test was not reported in almost half of the included studies. Also, studies that did report the interval had an interval of up to 7 days, which may be too late to detect thrombi, as there is evidence that cardiac thrombi may vanish within time, especially after administration of intravenous thrombolysis [29].

Four studies reported a time interval between tests of approximately 1 day [8, 15, 24, 27]. Of these studies, only one $(N=20)$ performed CTA on the same day as the ischemic stroke event and in this study CTA and TTE detected an equal amount of cardiac thrombi [8]. A minimal time interval between tests is important when comparing the two modalities, as especially the diagnostic yield of the second test that is performed can be negatively influenced due to this delay. In our systematic review, half of the studies performed echocardiography first and half CTA first.

Ambiguity remains regarding the additional value of ECG-gated imaging. Non-ECG gating is easier to implement and requires less expertise, however, ECG gating improves image quality of the moving heart. In this study, direct comparison of ECG gating versus non ECG gating was not possible due to the heterogeneity in study design, patient population and CT scanners. This also applies to the value of prospective versus retrospective ECG gating. Prospective ECG gating (or ECG triggering) allows for imaging of the heart in a specific point in the cardiac cycle (e.g. diastole, when the cardiac chambers can be optimally visualized) and results in less exposure to radiation than retrospective ECG gating. However, retrospective ECG gating (imaging of the heart throughout the cardiac cycle and selecting the desired images retrospectively) may increase the chance to acquire highquality images in patients with AF or other heart rhythm irregularities.

Overall, in our analysis CTA detected more thrombi than echocardiography and CTA had high sensitivity and specificity compared to TEE. Not surprisingly, the pooled sensitivity of CTA compared to TEE further increased when outdated scanners were excluded from the analysis. However, due to the great heterogeneity between studies, interpretation of the value of these results for everyday practice is difficult and should be done with caution. Studies that used older CT scanners, did not define thrombus on CTA, or performed CTA as second test are more likely to present false positive results. On the other hand, studies that used modern CT scanners, defined thrombus on CTA and echocardiography and performed CTA first are more likely to present true positive results. Interestingly, pooled sensitivity of CTA compared to TTE was low. This is possibly due to the fact that there was no patient level agreement on the thrombi that were found with both modalities. CTA did find more thrombi in total, but in some other patients TTE detected thrombi that were not found on CTA.

A recent review on cardiac CTA and TEE offers helpful advice and illustrative images on which technique to use in certain clinical settings [30], including cryptogenic stroke. A complicating factor is that criteria for cryptogenic stroke are not standardized. It may be worthwhile to study the diagnostic yield of cardiac CTA versus TEE in embolic stroke of undetermined source (ESUS). Per ESUS definition [3] CTA and TEE would be add-ons to TTE in this scenario. Notably, the recommendations in the review [30] are based in part on the aforementioned meta-analysis [28] of studies published before CTA from aortic arch to intracranial vessels became routine care for patient selection for endovascular treatment in most stroke centers. The review does not discuss the potential role of cardiac CTA as part of the acute phase work-up. A challenge in this context is that for effective implementation of heart-brain axis CTA in the acute phase of ischemic stroke in clinical practice it is paramount to establish interdisciplinary agreements for the 
responsibility and time frame for systematic reporting of the widened field, fitting with local preferences.

Although CTA may be a promising alternative to echocardiography for detection of cardiac thrombi and other highrisk cardio-aortic sources of embolism in ischemic stroke patients, it is unlikely CTA will fully replace echocardiography. Limitations of CTA include lack of dynamic heart function data, exposure to radiation and contrast media, suboptimal image quality in patients with tachycardia or arrhythmias and unsuitability as a bedside technique. Adjustment of the CTA protocol to include dynamic data is possible using retrospective ECG gating with cine sequences, but it results in increased exposure to radiation. Even though additional radiation associated with cardiac CTA is low if modern CT scanners are used [11], it remains a disadvantage particularly for young patients. Small studies have demonstrated it is feasible to perform non-ECG-gated heart-brain axis CTA in the acute phase of ischemic stroke quickly and without additional contrast media [8-10]. However, it is likely that ECG gating and cardiac contrast injection protocols increase image quality and diagnostic yield, resulting in longer acquisition times and increased risk of renal injury.

Though outside the scope of our study, a third technique with growing potential for imaging of the heart in stroke is magnetic resonance imaging (MRI) [31, 32]. Cardiac MRI is non-invasive and can provide functional and structural information. Main disadvantages include relatively high cost, less availability (especially in the acute phase of ischemic stroke), long acquisition times and lower spatial resolution compared with CTA.

The main limitation of this paper is the great heterogeneity between studies, missing data, and low quality of the included studies. Second, choosing diagnostic yield as our primary endpoint makes interpretation of disagreements in results between CTA and echocardiography challenging. This interpretation depends on factors such as the order in which tests were performed, time interval between tests and the type of CT scanner which was used. However, we believe that due to the lack of a true gold standard, presenting results in terms of sensitivity/specificity as primary endpoint might not do justice to the value of CTA, especially in studies in which echocardiography is more likely to present false negative results.

\section{Conclusion}

On the basis of our systematic review, CTA may be an attractive alternative to echocardiography for detection of cardiac thrombi in ischemic stroke patients. Heterogeneity between studies and the lack of an optimal gold standard complicate interpretation of diagnostic accuracy. Now that CTA of the cervical and intracranial arteries has become routine care to determine eligibility for endovascular treatment and additional radiation as a result of extending the CTA to include the heart is low with modern CT scanners, large multicenter prospective studies focusing on the diagnostic yield of cardiac CTA in the acute phase of ischemic stroke versus echocardiography (TTE and/or TEE) for detection of cardiac thrombi (and other high-risk cardio-aortic sources of embolism) are warranted. Another context worthwhile of study would be the diagnostic yield of cardiac CTA versus TEE in patients with ESUS.

Acknowledgements We thank Drs. René Spijker for his assistance in the literature search and Dr. Jan Willem van Dalen for his contribution to the statistical analyses.

Funding Drs. Guglielmi received research grants from the Royal Netherlands Academy of Arts and Sciences (Van Leersum Grant), Foundation De Drie Lichten, Remmert Adriaan Laan Fund, and AMC Young Talent Fund. The funders played no role in the preparation of the manuscript or the decision to submit for publication.

\section{Compliance with ethical standards}

Conflicts of interest Dr. Majoie reports grants from CVON/Dutch Heart Foundation, European Commission, TWIN Foundation, Dutch Health Evaluation program, and Stryker, outside the submitted work (paid to institution). Dr. Majoie and Dr. Roos are shareholders of Nico. lab, a company that focuses on the use of artificial intelligence for medical image analysis. All other authors declare no disclosures.

Ethical standards All studies in this review have been approved by the appropriate ethics committee and have therefore been performed in accordance with the ethical standards laid down in the 1964 Declaration of Helsinki and its later amendments.

Open Access This article is licensed under a Creative Commons Attribution 4.0 International License, which permits use, sharing, adaptation, distribution and reproduction in any medium or format, as long as you give appropriate credit to the original author(s) and the source, provide a link to the Creative Commons licence, and indicate if changes were made. The images or other third party material in this article are included in the article's Creative Commons licence, unless indicated otherwise in a credit line to the material. If material is not included in the article's Creative Commons licence and your intended use is not permitted by statutory regulation or exceeds the permitted use, you will need to obtain permission directly from the copyright holder. To view a copy of this licence, visit http://creativecommons.org/licenses/by/4.0/.

\section{References}

1. Kolominsky-Rabas PL, Weber M, Gefeller O, Neundoerfer B, Heuschmann PU (2001) Epidemiology of ischemic stroke subtypes according to TOAST criteria: incidence, recurrence, and long-term survival in ischemic stroke subtypes: a populationbased study. Stroke 32:2735-2740

2. Schulz UG, Rothwell PM (2003) Differences in vascular risk factors between etiological subtypes of ischemic stroke: importance of population-based studies. Stroke 34:2050-2059

3. Hart RG, Diener HC, Coutts SB, Easton JD, Granger CB, O’Donnell MJ, Sacco RL, Connolly SJ (2014) Embolic strokes 
of undetermined source: the case for a new clinical construct. Lancet Neurol 13:429-438

4. O'Carroll CB, Barrett KM (2017) Cardioembolic stroke. Continuum 23:111-132

5. Gladstone DJ, Spring M, Dorian P, Panzov V, Thorpe KE, Hall J, Vaid H, O'Donnell M, Laupacis A, Cote R, Sharma M, Blakely JA, Shuaib A, Hachinski V, Coutts SB, Sahlas DJ, Teal P, Yip S, Spence JD, Buck B, Verreault S, Casaubon LK, Penn A, Selchen D, Jin A, Howse D, Mehdiratta M, Boyle K, Aviv R, Kapral MK, Mamdani M, Investigators E (2014) Atrial fibrillation in patients with cryptogenic stroke. N Engl J Med 370:2467-2477

6. Saric M, Armour AC, Arnaout MS, Chaudhry FA, Grimm RA, Kronzon I, Landeck BF, Maganti K, Michelena HI, Tolstrup K (2016) Guidelines for the use of echocardiography in the evaluation of a cardiac source of embolism. J Am Soc Echocardiogr 29:1-42

7. Pepi M, Evangelista A, Nihoyannopoulos P, Flachskampf FA, Athanassopoulos G, Colonna P, Habib G, Ringelstein EB, Sicari R, Zamorano JL, Sitges M, Caso P (2010) Recommendations for echocardiography use in the diagnosis and management of cardiac sources of embolism: European Association of Echocardiography (EAE) (a registered branch of the ESC). Eur J Echocardiogr 11:461-476

8. Yeo LLL, Holmin S, Andersson T, Lundstrom E, Gopinathan A, Lim EL, Leong BSH, Kuan WS, Ting E, Tan BYQ, Eide SE, Tay ELK (2017) Nongated cardiac computed tomographic angiograms for detection of embolic sources in acute ischemic stroke. Stroke 48:1256-1261

9. Popkirov S, Schlegel U, Weber W, Kleffner I, Altenbernd J (2019) Cardiac imaging within emergency CT angiography for acute stroke can detect atrial clots. Front Neurol 10:349

10. Guglielmi V, Planken RN, Mihl C, Niesen S, Staals J, Coutinho JM, Postma AA (2020) Non-gated cardiac CT angiography for detection of cardio-aortic sources of embolism in the acute phase of ischaemic stroke. J Neurol Neurosurg Psychiatry. https://doi. org/10.1136/jnnp-2019-321923

11. Faletra FF, D’Angeli I, Klersy C, Averaimo M, Klimusina J, Pasotti E, Pedrazzini GB, Curti M, Carraro C, Diliberto R, Moccetti T, Auricchio A (2010) Estimates of lifetime attributable risk of cancer after a single radiation exposure from 64-slice computed tomographic coronary angiography. Heart 96:927-932

12. Ouzzani M, Hammady H, Fedorowicz Z, Elmagarmid A (2016) Rayyan - a web and mobile app for systematic reviews. Syst Rev 5:210

13. McInnes MDF, Moher D, Thombs BD, McGrath TA, Bossuyt PM, Clifford T, Cohen JF, Deeks JJ, Gatsonis C, Hooft L, Hunt HA, Hyde CJ, Korevaar DA, Leeflang MMG, Macaskill P, Reitsma JB, Rodin R, Rutjes AWS, Salameh JP, Stevens A, Takwoingi Y, Tonelli M, Weeks L, Whiting P, Willis BH (2018) Preferred reporting items for a systematic review and meta-analysis of diagnostic test accuracy studies: the PRISMA-DTA statement. JAMA 319:388-396

14. Whiting PF, Rutjes AW, Westwood ME, Mallett S, Deeks JJ, Reitsma JB, Leeflang MM, Sterne JA, Bossuyt PM (2011) QUADAS-2: a revised tool for the quality assessment of diagnostic accuracy studies. Ann Intern Med 155:529-536

15. Helgason CM, Chomka E, Louie E, Rich S, Zajac E, Roig E, Wilbur A, Brundage BH (1989) The potential role for ultrafast cardiac computed tomography in patients with stroke. Stroke 20:465-472

16. Love BB, Struck LK, Stanford W, Biller J, Kerber R, Marcus M (1990) Comparison of two-dimensional echocardiography and ultrafast cardiac computed tomography for evaluating intracardiac thrombi in cerebral ischemia. Stroke 21:1033-1038

17. Hur J, Kim YJ, Nam JE, Choe KO, Choi EY, Shim CY, Choi BW (2008) Thrombus in the left atrial appendage in stroke patients: detection with cardiac CT angiography-a preliminary report. Radiology 249:81-87

18. Hur J, Kim YJ, Lee HJ, Ha JW, Heo JH, Choi EY, Shim CY, Kim TH, Nam JE, Choe KO, Choi BW (2009) Cardiac computed tomographic angiography for detection of cardiac sources of embolism in stroke patients. Stroke 40:2073-2078

19. Boussel L, Cakmak S, Wintermark M, Nighoghossian N, Loffroy R, Coulon P, Derex L, Cho TH, Douek PC (2011) Ischemic stroke: etiologic work-up with multidetector $\mathrm{CT}$ of heart and extra- and intracranial arteries. Radiology 258:206-212

20. Kim SC, Chun EJ, Choi SI, Lee SJ, Chang HJ, Han MK, Bae HJ, Park JH (2010) Differentiation between spontaneous echocardiographic contrast and left atrial appendage thrombus in patients with suspected embolic stroke using two-phase multidetector computed tomography. Am J Cardiol 106:1174-1181

21. Ko SB, Choi SI, Chun EJ, Ko Y, Park JH, Lee SJ, Lee J, Han MK, Bae HJ (2010) Role of cardiac multidetector computed tomography in acute ischemic stroke: a preliminary report. Cerebrovasc Dis 29:313-320

22. Hur J, Kim YJ, Lee HJ, Nam JE, Ha JW, Heo JH, Chang HJ, Kim HS, Hong YJ, Kim HY, Choe KO, Choi BW (2011) Dualenhanced cardiac CT for detection of left atrial appendage thrombus in patients with stroke: a prospective comparison study with transesophageal echocardiography. Stroke 42:2471-2477

23. Kim SJ, Choe YH, Park SJ, Kim GM, Chung CS, Lee KH, Bang OY (2012) Routine cardiac evaluation in patients with ischaemic stroke and absence of known atrial fibrillation or coronary heart disease: transthoracic echocardiography vs. multidetector cardiac computed tomography. Eur J Neurol 19:317-323

24. Sipola P, Hedman M, Onatsu J, Turpeinen A, Halinen M, Jakala P, Vanninen R (2013) Computed tomography and echocardiography together reveal more high-risk findings than echocardiography alone in the diagnostics of stroke etiology. Cerebrovasc Dis 35:521-530

25. Lee K, Hur J, Hong SR, Suh YJ, Im DJ, Kim YJ, Hong YJ, Lee HJ, Kim YJ, Lee HS, Hong GR, Choi BW (2015) Predictors of recurrent stroke in patients with ischemic stroke: comparison study between transesophageal echocardiography and cardiac CT. Radiology 276:381-389

26. Ajlan AM, Bagdadi RR, Alama MN, Ayoub O (2016) Impact of implementing cardiac CT in evaluating patients suspected of cardioembolic stroke. J Comput Assist Tomogr 40:380-386

27. Taina M, Vanninen R, Sipola P, Muuronen A, Jakala P, Hedman M (2016) Cardiac CT differentiates left atrial appendage thrombi from circulatory stasis in acute stroke patients. Vivo 30:671-676

28. Romero J, Husain SA, Kelesidis I, Sanz J, Medina HM, Garcia MJ (2013) Detection of left atrial appendage thrombus by cardiac computed tomography in patients with atrial fibrillation: a metaanalysis. Circ Cardiovasc Imaging 6:185-194

29. Ferrari E, Benhamou M, Berthier F, Baudouy M (2005) Mobile thrombi of the right heart in pulmonary embolism: delayed disappearance after thrombolytic treatment. Chest 127:1051-1053

30. Pathan F, Hecht H, Narula J, Marwick TH (2018) Roles of transesophageal echocardiography and cardiac computed tomography for evaluation of left atrial thrombus and associated pathology: a review and critical analysis. JACC Cardiovasc Imaging 11:616-627

31. Pagan RJ, Parikh PP, Mergo PJ, Gerber TC, Mankad R, Freeman WD, Shapiro BP (2015) Emerging role of cardiovascular $\mathrm{CT}$ and MRI in the evaluation of stroke. AJR Am J Roentgenol 204:269-280

32. Yaghi S, Liberman AL, Atalay M, Song C, Furie KL, Kamel H, Bernstein RA (2017) Cardiac magnetic resonance imaging: a new tool to identify cardioaortic sources in ischaemic stroke. J Neurol Neurosurg Psychiatry 88:31-37 